\section{Antikörper wirkt bei hartnäckiger Neurodermitis}

\author{
Inwieweit Dupilumab in der Lage ist, hartnäckige Neurodermitis-Symptome \\ zu bessern, wurde in einer Dosisfindungsstudie getestet.
}

$\mathrm{D}$ upilumab ist ein monoklonaler Antikörper, der am Interleukin-4-Rezeptor $\alpha$ angreift. Damit blockiert er die Aktivierung der Interleukine 4 und 13, die in der Pathogenese der atopischen Dermatitis eine wichtige Rolle spielen.

Wirksamkeit und Sicherheit mehrerer Dupilumab-Regime bei Neurodermitis wurden jetzt in einer randomisierten, kontrollierten Phase-IIb-Studie getestet. Bei den Teilnehmern handelte es sich um erwachsene Neurodermitispatienten, die unter mittelgradigen bis schweren Symptomen litten, die auf eine topische Therapie innerhalb eines halben Jahres nur unzureichend angesprochen hatten.

Die 379 Patienten wurden nach dem Zufallsprinzip in sechs Gruppen eingeteilt: Fünf erhielten unterschiedliche Antikörper-Regime, jeweils s.c. Die sechste Gruppe (61 Patienten) bekam ein Placebo gespritzt. Als primären Endpunkt hatten die Forscher die prozentuale Veränderung im EASI-Score innerhalb von 16 Wochen nach Therapiebeginn definiert.
In nahezu allen Verumgruppen zeigte sich eine deutliche dosisabhängige Verbesserung im Vergleich zu Placebo. Die Ergebnisse für die einzelnen Regime: _300 mg einmal die Woche: $-73,7 \%$ _300 mg alle zwei Wochen: $-68,2 \%$ _200 mg alle zwei Wochen: $-65,4 \%$ -300 mg alle vier Wochen: $-63,5 \%$ $-100 \mathrm{mg}$ alle vier Wochen: $-44,8 \%$. Unter Placebo gingen die Werte im EASIScore im Mittel um 18,1 \% zurück. Damit waren die Unterschiede zu Placebo für alle 300- und 200-mg-Regime signifikant; lediglich das 100-mg-Regime erzielte kein signifikantes Ergebnis.

Deutlich mehr Patienten erreichten unter Dupilumab einen IGA-Wert von 0 oder 1, was einer (fast) ekzemfreien Haut entspricht. Und auch im SCORAD-Score (SCORing Atopic Dermatitis), der Intensität und Ausmaß der Hautveränderungen misst, waren die Verumgruppen jeweils Placebo deutlich überlegen, mit Ausnahme der 100-mg-Gruppe. Signifikante Verbesserungen ergaben sich (au-
Ber bei $100 \mathrm{mg}$ ) auch bei den subjektiven Kriterien Juckreiz (PNRS) und Lebensqualität (DLQI).

In den meisten Fällen hatte sich die Krankheitsaktivität bereits nach einer Woche deutlich gebessert. Obwohl der Antikörper insgesamt gut toleriert wurde, brachen 21 Patienten die Therapie vorzeitig ab. Zehn von ihnen stammten aus der 100-mg-Gruppe. Auffällig waren 13 Fälle mit Lippenherpes, von denen sich die meisten ebenfalls in dieser Gruppe fanden, und ein Asthmafall.

Fazit: Wie die Phase-IIb-Studie nahelegt, lassen sich mittelschwere bis schwere Neurodermitis-Symptome durch Dupilumab signifikant bessern. Insbesondere der Juckreiz ließ sich bei ausreichender Dosierung gut unter Kontrolle bringen. Weitere Studien müssen unter anderem klären, ob die Wirkung und Sicherheit auch bei längerer Anwendung und in Kombination mit einer Lokaltherapie erhalten bleibt und was es mit dem Lippenherpes auf sich hat. Dr. Elke Oberhofer

Thaçi $D$ et al. Efficacy and safety of dupilumab in adults with moderate-to-severe atopic dermatitis inadequately controlled by topical treatments: a randomised, placebo-controlled, doseranging phase $2 \mathrm{~b}$ trial. Lancet 2016; 387: 40-52

\title{
Nasenfilter schützt vor Rhinitissymptomen
}

\section{Pollenundurchlässige Nasenfilter bieten einen kausalen Therapieansatz bei saisonaler allergischer Rhinitis. Eine Proof-of-Concept-Studie zur Effektivität, Verträglichkeit und Akzeptanz verlief zufriedenstellend.}

$\mathrm{M}$ echanische Schutzsysteme zur Vermeidung des Allergenkontakts mit der Nasenschleimhaut, bisher überwiegend in Form von Atemmasken oder Nasensalben angeboten, haben sich bei Patienten mit saisonaler allergischer Rhinitis nicht recht durchsetzen können. Neue, intranasale Nasenfilter könnten das ändern. Sie stören die Träger offensichtlich kaum und erwiesen sich in ersten Untersuchungen in Pollenkammerversuchen als brauchbar. Jetzt stand die Erprobung unter natürlichen Bedingungen während der Pollensaison an. Man bediente sich dabei einer „Tag-im-ParkStudie“. Anders als bei multizentrischen
Studien über die gesamte Pollensaison erlauben Parkstudien eine genauere Assoziation zwischen Pollenbelastung und Symptomatik.

Einbezogen in die monozentrische, doppelblinde, placebokontrollierte Studie waren 65 erwachsene Gräserpollenallergiker, die entweder die neuen $\mathrm{Na}$ senfilter oder Placebofilter über zwei Tage im Cross-over-Design während der Hauptgräserpollensaison trugen. Das Einsetzen der Filter sollte möglichst bei noch geringer Symptombelastung erfolgen. Primäres Studienziel war der Unterschied zwischen beiden Gruppen beim nasalen Gesamtsymptomenscore (TNSS) mit insgesamt 13 Einzelitems. Hier ergab sich ein signifikanter Unterschied zugunsten der Nasenfilter $(p=0,03$ ), auch der Unterschied bei den Maximal-TNSS zwischen beiden Gruppen war signifikant $(p=0,03)$, entsprechend einem relativen Rückgang der Beschwerden von $40 \%$ und $43 \%$. Überdurchschnittlich gut schützten die Filter vor Niesen und laufender Nase. Sogar das Augentränen ließ bei den Probanden mit Verumfilter deutlich nach. Schwerwiegende Nebenwirkungen wurden nicht beschrieben, die Akzeptanz war gut.

Fazit: Ein neuer Nasenfilter reduzierte im Vergleich zu einem Placebofilter die Symptome einer allergischen Gräserpollenrhinitis signifikant. Dr. Barbara Kreutzkamp

Kenney et al. Preventive effect of nasal filters on allergic rhinitis: A randomized, double-blind, placebo-controlled crossover park study.

J Allergy Clin Immunol 2015; 136: 1566-72 